\title{
Laparoscopic Management of a Spontaneous Interstitial Heterotopic Pregnancy with Preservation of the Intrauterine Pregnancy: Case Report from Middle East
}

\author{
Ahmad Talal Chamsi* and Ashraf Dawood \\ Department of Obstetrics and Gynecology, Women Specialized Hospital, King Fahad Medical City, Riyadh, Saudi Arabia
}

${ }^{*}$ Corresponding author: Ahmad Talal Chamsi, Department of Obstetrics and Gynecology, Women Specialized Hospital, King Fahad Medical City, P.O. Box 3643, Riyadh 11481, Saudi Arabia, Tel: +966 554070709; Fax: +966 11 8026389; Email: ahmadchamsi@hotmail.com

Rec date: Mar 01, 2018; Acc date: Mar 23, 2018; Pub date: Mar 27, 2018

Citation: Chamsi, AT, Dawood A (2018) Laparoscopic Management of a Spontaneous Interstitial Heterotopic Pregnancy with Preservation of the Intrauterine Pregnancy: Case Report from Middle East. Gynecol Obstet Case Rep Vol.4: No.1: 64.

\section{Abstract}

\begin{abstract}
Heterotopic pregnancy is defined as multiple gestation in which one gestational sac is intrauterine while the other is extra uterine- most commonly tubal ectopic pregnancy. An interstitial ectopic pregnancy is one of the most lifethreatening types of ectopic gestations, with a mortality rate that can reach 6-7 times higher than that of the ectopic pregnancies in general. In our case report, heterotopic pregnancy occurs after a spontaneous conception cycle. A 34-year-old Gravid 3 Para 1 + Abortion 1 woman with a gestational age of 8 weeks +3 days by LMP. She was diagnosed and managed laparoscopicaly by right salpingectomy with preservation of intrauterine pregnancy. She was discharged on the second postoperative day and followed-up regularly at the obstetrics clinic with uneventful antenatal follow up. This might be the first case to be presented from Middle east with such scenario. Careful evaluation of the adnexa is mandatory not only in women undergoing assisted reproduction, but also with spontaneous pregnancy to avoid delayed diagnosis in such cases.
\end{abstract}

Keywords: Heterotopic pregnancy; First trimester; Ruptured; Laparoscopy; Ectopic

\section{Introduction}

Heterotopic pregnancy is defined as multiple gestation in which one gestational sac is intrauterine while the other is extra uterine- most commonly tubal ectopic pregnancy. It was first reported as an autopsy finding in 1708 [1]. Heterotopic pregnancy is a live threatening condition especially that it is often diagnosed later after tubal rupture has already occurred [2]. Heterotopic pregnancies are very rare occurrence in spontaneous pregnancies with an incidence of approximately $1 / 30000(1 / 10000$ to $1 / 50000)[3,4]$, but its overall incidence has increased with the use of assisted reproductive techniques (ART), in which cases the incidence is ranging from $1 / 100$ to $1 / 360$ [3]. The most common site for an ectopic pregnancy is the ampullary part of the fallopian tube, representing $70 \%$ of the ectopic pregnancies, then Fimbrial (12\%), Isthmic (11\%), interstitial (2-3\%), Ovarian (1\%), Scar ectopic (1\%), cervical and abdominal ectopic (1\%) [1]. An interstitial ectopic pregnancy is one of the most life-threatening types of ectopic gestations, with a mortality rate that can reach 6-7 times higher than that of the ectopic pregnancies in general [5]. We hereby present a case of intestinal heterotopic pregnancy that occurred after a spontaneous conception cycle. This might be the first case ever presented with such a scenario from the middle east. She presented with amenorrhea for eight weeks and acute abdomen. Patient was diagnosed with laparoscopy as ruptured interstitial pregnancy and managed laparoscopically with the preservation of the intrauterine gestational pregnancy.

\section{Case Report}

A 34-year old Gravid 3 Para $1+$ Abortion 1 woman with a gestational age of 8 weeks +3 days by LMP, presented to Emergency Department of Women Specialized Hospital, King Fahad Medical City, Riyadh complaining of Lower Abdominal pain, mainly in the right lower quadrant. The pain was sharp in nature, duration started few hours prior to arrival our Emergency Department (ED). Increasing in severity, reaching $10 / 10$ on Wong-Baker FACES Pain Rating Scale. Pain was not relieved by analgesics and associated with dizziness. Regarding obstetrical history, she is married for 9 years with a previous Cesarean delivery due to fetal distress 7 years back followed by one first trimester spontaneous miscarriage. She has a regular menstrual cycle. This pregnancy was planned. No history of pelvic inflammatory disease and gave history of using combined contraceptive pills that was stopped 6 months ago. She is known case of hypothyroidism on levothyroxine. She has an unremarkable family history and not allergic to any food or drug. Patient presented to ED earlier with similar complaint and was discharged home because she was stable vitally and her exam was unremarkable and 1-gram IV paracetamol relived all her symptoms. Bedside Ultrasound confirming and intrauterine pregnancy with no abnormalities identified (Figure 1). Few hours later presented again increased abdominal pain. Patient was conscious alert and oriented, afebrile with Blood Pressure of 122/70 millimeters of mercury, 
Heart Rate of 117 beat per minute, respiratory rate of 18 breath per minute and Oxygen saturation of $100 \%$ on Room Air. Abdominal examination revealed diffuse, lower abdominal tenderness with guarding and rebound tenderness mainly in the right lower quadrant suggesting an acute abdomen. Complete Blood Count revealed a hemoglobin of $11 \mathrm{~g} / \mathrm{dl}$ and white blood cell of $20.20 \times 109 /$ L. Abdominal Ultrasound was done to rule out appendicitis, cholecystits, or pyelonephritis, as heterotopic pregnancy was not suspected due to the presence of viable intra uterine pregnancy by the earlier ultrasound done on the same day. The abdominal ultrasound revealed no evidence of cholecystitis, pyelonephritis or appendicitis with fluid collection in the pelvis and abdomen. A trial to repeat the Bedside scan failed due to increase in the pain. Per speculum examination showed positive cervical motion tenderness but no vaginal loss with. Plan was to do Emergency diagnostic laparoscopy. Operative finding revealed a ruptured ectopic pregnancy and the interstitial part of the right fallopian tube with Haemoperitoneum of $800 \mathrm{ml}$ reaching the peri-hepatic region (Figure 2). Right salpingectomy was performed using electrosurgery followed by removal of the haemoperitoneum and free clots. She had a preoperative Hemoglobin of $11 \mathrm{~g} / \mathrm{dl}$ and intraoperative Hemoglobin of 7.6 $\mathrm{g} / \mathrm{dl}$ with otherwise normal coagulation profile, liver function and renal function tests. Patient had a good recovery period and received a total of 2 units packed red blood cells. A transvaginal ultrasound scan done postoperative day 1 revealed a viable intrauterine pregnancy going with date.

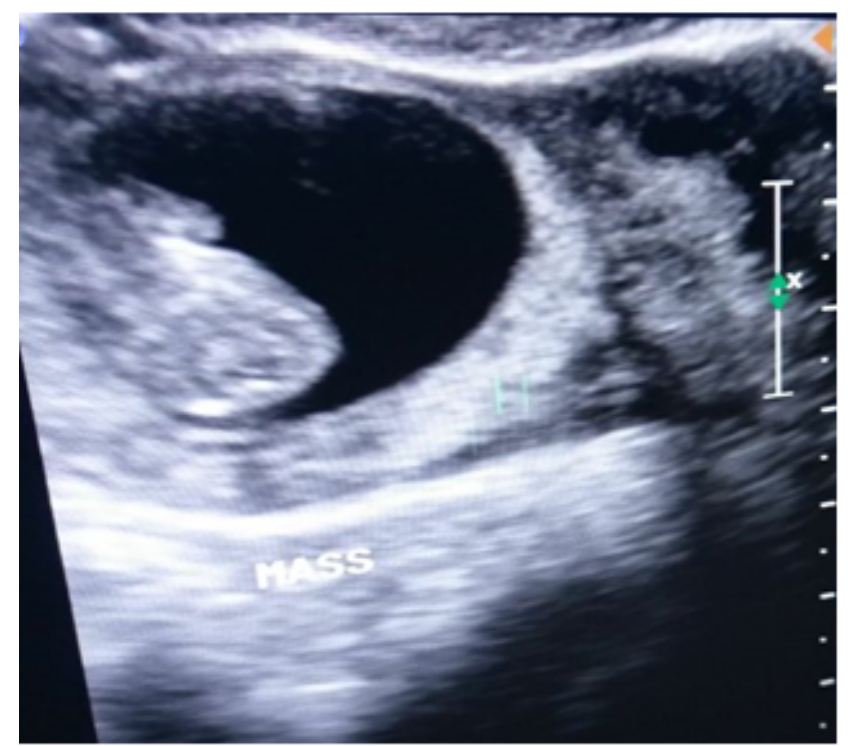

Figure 1 Transabdominal ultrasound of the uterus (transverse plane) showing intrauterine gestational sac.

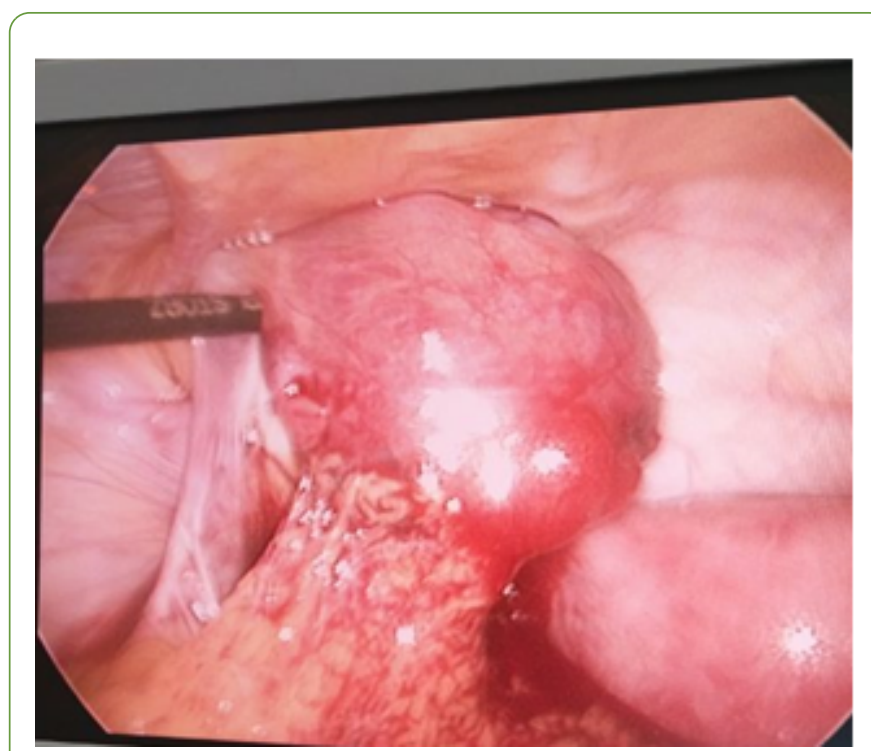

Figure 2 Intraoperative image showing the uterus with a ruptured right interstitial pregnancy.

She was discharged on the second postoperative day and followed-up regularly at the obstetrics clinic with double iron. Her hemoglobin went back to normal values of $12.2 \mathrm{~g} / \mathrm{dl}$ during the follow up period. The intraoperative specimen was followed up and confirmed a right ectopic pregnancy. Patient had later on multiple antenatal visits that were all reassuring. She underwent an Anatomy scan that was reassuring as well. She is doing well with her pregnancy up to the moment this report is being written.

\section{Discussion}

Heterotopic pregnancy (HP), might be a fatal obstetric complication to both mother and intrauterine pregnancy. Because of the very low incidence of HP, the literature is lacking evidence-based recommendations, and the majority of the current practice is based on case reports and expertise opinion. The majority of HP cases diagnosed late and hence significant morbidity and occasional mortality have been reported as a result of a delay in diagnosis [6]. Therefore, it is very important to be ruled out in any female patient with abdominal pain in the first trimesters and especially after assisted reproductive technology pregnancy. A systematic review carried out by Talbot [7] from 2005 to 2010 showed that in $33 \%$ of Heterotopic pregnancies the diagnosis was delayed because previous sonographic findings of a normal intrauterine pregnancy gave false reassurance. Therefore, this high rate of misdiagnosis emphasizes the high importance of a complete evaluation of the adnexae when performing a first trimester pregnancy ultrasound. The diagnosis in our case was difficult to establish because of false reassurance that was obtained by the first ultrasound that revealed an intrauterine pregnancy and patient presentation with leukocytosis that has given the impression of non gynecological cause like acute appendicitis or cholecystitis and the delay afterward for obtaining an abdominal ultrasound that revealed the fluid 
collection. Both laparotomy and laparoscopy appear to be safe and with equal results regarding the intrauterine pregnancy [8]. In our case a laparoscopy was preferred because of the skilled staff availability.

\section{Conclusion}

Diagnosis may be further delayed by a lack of symptoms. In a series of 13 women with heterotopic pregnancy who had undergone different types of fertility assistance, 7 (54\%) of the patients were asymptomatic [9]. The mean gestational age at time of diagnosis was eight weeks, consistent with other case series and with our case [10]. The case presented here is interesting in terms of its rarity- interstitial heterotopic pregnancy following spontaneous conception in the absence of any other risk factors, and successful preservation of the intrauterine pregnancy despite delayed diagnosis of the heterotopic gestation. Laparoscopic management is successful even in the presence of large hemoperitoneum and is preferred to laparotomy. This might be the first case to be presented from Middle east with such scenario. Careful evaluation of the adnexa is mandatory not only in women undergoing assisted reproduction, but also with spontaneous pregnancy.

\section{Ethical Approval}

Given the nature of the article, the management of the patient was not modified by the study, so it was considered exempted from IRB approval.

Patient had an informed consent for the management and permission for using the data for research study if needed.

\section{Conflict of Interests and Funding Sources Statement}

None

\section{Acknowledgment}

None

\section{Authors' Contributions}

Ahmed Talal Chamsi manuscript development, literature review, medical and surgical management of the patient.

Ashraf Dawood manuscript review, literature review, medical and surgical management of the patient. All authors have read and approved the final draft of manuscript.

\section{References}

1. Bright DA, Gaupp FB (1990) Heterotopic pregnancy: a reevaluation. J Am Board Fam Pract 3: 125-128.

2. Lund PR, Sielaff GW, Aiman EJ (1989) In vitro fertilization patient presenting in hemorrhagic shock caused by unsuspected heterotopic pregnancy. Am J Emerg Med 7: 49-53.

3. Basile F, Di Cesare C, Quagliozzi L, Donati L, Bracaglia M, et al. (2012) Spontaneous heterotopic pregnancy, simultaneous ovarian, and intrauterine: a case report, Case Reports. Obstetrics and Gynecology 2012: 509694.

4. Sun SY, Araujo Júnior E, Elito Júnior J, Rolo LC, Campanharo FF, et al. (2012) Diagnosis of heterotopic pregnancy using ultrasound and magnetic resonance imaging in the first trimester of pregnancy: a case report. Case Rep Radiol 2012: 317592.

5. Shan N, Dong D, Deng W, Fu Y (2014) Unusual ectopic pregnancies: a retrospective analysis of 65 cases. J Obstet Gynaecol Res 40: 1466

6. Mistry BM, Balasubramaniam S, Silverman R, Sakabu SA, Troop BR (2000) Heterotopic pregnancy presenting as an acute abdomen: a diagnostic masquerader. Am Surg 66: 307e308.

7. Talbot K, Simpson R, Price N, Jackson SR (2011) Heterotopic pregnancy. J Obstet Gynaecol 31: 7-12.

8. Tandon R, Goel P, Saha PK (2009) Spontaneous heterotopic pregnancy with tubal rupture: a case report and review of the literature. J Med Case Rep 3: 8153.

9. Louis-Sylvestre C, Morice P, Chapron C, Dubuisson JB (1997) The role of laparoscopy in the diagnosis and management of heterotopic pregnancies. Hum Reprod 12: 1100-1102.

10. Wang LL, Chen X, Ye DS, Liu YD, He YX, et al. (2014) Misdiagnosis and delayed diagnosis for ectopic and heterotopic pregnancies after in vitro fertilization and embryo transfer. J Huazhong Univ Sci Technolog Med Sci 34: 103-107. 